\title{
Efficacy of Zinc Supplement in Minimal hepatic Encephalopathy: A prospective, Randomized Controlled Study (Zinc-MHE Trial)
}

\author{
Rattaya Janyajirawong ${ }^{1}$, Ratha-Korn Vilaichone ${ }^{2,3}$, Supatsri Sethasine ${ }^{1 *}$
}

\begin{abstract}
Background: Minimal hepatic encephalopathy (MHE) in patients with cirrhosis of the liver has a negative impact on the quality of daily life by impairing attention, memory and visuomotor coordination, and resulting in cognitive decline. Ammonia is thought to be part of the pathogenesis of hepatic encephalopathy. Zinc is an essential trace element, one of the cofactor enzymes that is essential for the conversion of ammonia to urea. Aim: To assess the effect of zinc supplementation on psychomotor performance in cirrhotic patients with MHE. Methods: This prospective, randomized, controlled trial recruited 69 cirrhotic patients (age 18-75 years) diagnosed with MHE by neuropsychometric (NP) tests comprised of the number connection test part A (NCT-A), number connection test part B (NCT-B), serial dot test (SDT), line tracing test (LTT) and digit symbol test (DST). Eligible patients were randomly assigned (1:1) by a computer-based system block of four randomizations to receive $45 \mathrm{mg}$ of elemental zinc or placebo for 12 weeks. The primary endpoint was the absolute change in NP tests from baseline to 12-weeks of zinc supplement compared with placebo. The assessment of changes of the health-related quality of life (HRQOL) using the Short Form survey-36 (SF-36) questionnaire, as well as biochemical parameters including serum ammonia, was also conducted in both groups. Results: From January to December 2020, 125 eligible cirrhotic patients were diagnosed with liver cirrhosis, of whom $69(55 \%)$ had MHE and were randomly assigned to treatment: 35 patients were assigned to receive $45 \mathrm{mg}$ of elemental zinc and the others 34 patients to receive placebo. Significant improvements in NP tests were established in the zinc supplement group when compared with the placebo group (NCT-A, $p=0.029$; NCT-B, $p=0.008$; SDT, $p=0.002$; DST, $p=<0.001)$. A significant improvement of HRQOL assessed by the SF-36 score was only seen in the zinc group $(\mathrm{p}<0.001)$. In the zinc supplement group, not only was an improvement in psychomotor performance reported, but quality of life was also improved, irrespective of baseline zinc level. Conclusion: Twelve weeks of zinc supplement in cirrhotic patients with MHE not only had a positive effect on psychomotor performance but also improved HRQOL irrespective to baseline zinc level.
\end{abstract}

Keywords: Zinc supplement- hepatic encephalopathy- quality of life (QoL)

Asian Pac J Cancer Prev, 22 (9), 2879-2887

\section{Introduction}

The major complications of cirrhosis include hepatic encephalopathy (HE), ascites and bleeding of the varicea, with hepatocellular carcinoma (HCC) representing the end of the spectrum of chronic liver diseases, independent of etiology.

Hepatic encephalopathy, one of the common complications of cirrhosis, manifests in neuropsychiatric problems. Minimal hepatic encephalopathy (MHE) is the reticence form of HE. The prevalence of MHE is approximately $40-60 \%$ of the cirrhotic patients (Maldonado-Garza et al., 2011; Rathi et al., 2019; Elgohary et al., 2020; Das et al., 2001).MHE is not detected by clinical and physical examination or laboratory investigation, but is only detected by neuropsychometric (NP) tests or neurophysiological tests (Vilstrup et al., 2014).

For NP tests, the Psychometric Hepatic Encephalopathy Score (PHES) is the sum score achieved from five psychometric tests comprised of the number connection test part A (NCT-A), number connection test part B (NCT-B), digit symbol test (DST), serial dotting test (SDT) and line tracing test (LTT) (Weissenborn, 2015). A diagnosis of MHE is appropriate if the PHES sum scores is less than -4 points (Weissenborn, 2008).

MHE has a negative impact on declines in various functions of patients by impairing work performance,

${ }^{1}$ Division of Gastroenterology and Hepatology, Department of Medicine, Faculty of Medicine, Vajira Hospital, Navamindradhiraj University, Bangkok, Thailand. ${ }^{2}$ Excellence Center in Digestive Diseases, Faculty of Medicine and Chulabhorn International College of Medicine (CICM), Thammasat University, Pathumthani, Thailand. ${ }^{3}$ Division of Gastroentero-Hepatology, Department of Internal Medicine, Faculty of Medicine, Universitas Airlangga, Surabaya, Indonesia.*For Correspondence: supatsri9@hotmail.com 
driving capacity and impaired cognitive function (Felipo et al., 2013; García-García et al., 2018; Urios et al., 2017, Bajaj et al., 2020; Agrawal et al., 2015; Bajaj et al.,2008). Furthermore, MHE not only increases the risk of proceeding to overt hepatic encephalopathy but is also associated with the progression of cirrhosis (Ampuero et al., 2018).

Although the pathogenesis of HE is not well clarified, ammonia is an important biochemical substance in the pathogenesis of HE (Shawcross and Jalan, 2005; Ciećko-Michalska et al., 2012). Zinc is an essential cofactor of ornithine transcarbamylase enzymes which enhance the conversion of ammonia to urea and promote glutamine synthetase for the metabolism of ammonia to glutamine in the skeletal muscle. Zinc supplementation can improve health-related quality of life (HRQOL) by decrement of serum ammonia (Marchesini et al., 1996; Katayama,2020). The aim of the study was to assess the effect of zinc supplementation on psychomotor performance in cirrhotic patients with MHE.

\section{Materials and Methods}

In this prospective, randomized, controlled trial, patients with cirrhosis were recruited at the liver clinic, department of medicine, Vajira hospital in Thailand From January to December 2020. The diagnosis of cirrhosis was based on clinical and laboratory tests, and imaging by ultrasonography or computed tomography of the abdomen.

\section{Inclusion criteria}

Age between 18-75 years at time of screening. Diagnosis of cirrhosis was confirmed by liver function test and ultrasound. Eligible cirrhotic patients were screened for an MHE diagnosis by NP tests comprised of five tests including NCT-A, NCT-B, SDT, LTT and DST. Diagnosis of MHE was confirmed if a summation score was less than -4 points (Weissenborn, 2008; Padilla, 2016; Li et al., 2013; Shiha and Mousa, 2019; Ware et al., 2000).

\section{NCT-A}

For testing psychomotor speed, there were 25 sequentially numbered circles on a sheet of paper. Patients were timed while drawing a continuous line from the smallest to the largest number. If an error was made, the patient had to correct it without stopping the timer. Each patient was assigned a total time for the time it took to draw a line from number 1 to number 25 inclusive of error correction time. A shorter time to complete the test indicated better performance.

\section{NCT-B}

For testing psychomotor speed, attention and mental tracking, there were 25 circles, thirteen of which were sequentially numbered from 1 to 13 , while the other twelve were labeled with the first 12 characters of the Thai alphabet. Patients had to draw a line which alternated from the lowest remaining number to the earlier remaining alphabet character. If an error was made, the patient had to correct without stopping the timer. The time it took each patient to draw the line correctly was evaluated. A shorter time to complete the test indicated better performance.

\section{$S D T$}

For testing psychomotor speed, patients had to draw a dot in the middle of 100 circles then time spent was evaluated. A shorter time to complete the test indicated better performance.

\section{$L T T$}

For testing psychomotor speed and visuomotor ability, patients had to draw a continuous line between two parallel lines without intersecting either of the two given parallel lines. Total time spent was evaluated. A shorter time to complete the test indicated better performance.

\section{$D S T$}

For testing psychomotor speed and attention patients were shown a group squares with a number in each square and a symbol underneath each number. Patients had to remember and add symbols according to the numbers in the given squares. The test result was evaluated by the number of fields a patient could fill in without any false or omitted fields in 90 seconds. A higher score indicated good performance.

All of the cirrhotic patients with MHE self-administered a 36-item Short Form Survey (SF-36) questionnaire in Thai answering questions about their health-related quality of life (HRQOL). The SF-36 consisted of eight aspects of health totaling 35 questions: assessing 10 questions of physical functioning (PF), 4 questions of role physical (RP), 2 questions of bodily pain (BP), 5 questions of perception health (PH), 4 questions of vitality (VT), 2 questions of social functioning (SF), 3 questions of role emotional (RE) and 5 questions of mental health (MH). The 36th question, which asks about change in health in one year, was not included in the scale or summary scores. Score from each aspect were from 0 to 100 , higher scores indicated better health (Ware et al., 2000).

\section{Exclusion criteria}

Patients with a history of overt HE, gastrointestinal bleeding and spontaneous bacterial peritonitis in the past 6 weeks, neurological diseases caused by impaired cognitive function such as epilepsy, Alzheimer's disease and dementia, recent alcohol consumption within 6 weeks, and medication affecting psychometric competency like benzodiazepines or antidepressant drugs, concurrent with hepatocellular carcinoma or active other malignancy, pregnancy or blindness.

\section{Randomization and masking}

Eligible patients were randomly assigned to $1: 1$ ratio to receive oral zinc or placebo. Randomization was performed using a computer-based system, with a permuted block of four. Zinc supplements present as zinc amino acid (bisglycinate) chelate tablets $(75 \mathrm{mg}$ per tablet) consist of elemental zinc ( $15 \mathrm{mg}$ per tablet), supplemented with $45 \mathrm{mg}$ of elemental zinc per day. Zinc and placebo were provided as tablets in identical containers labelled with coded colors. Both physicians and participants were blinded to the treatment assignment. Blood samples were 
obtained to evaluate complete blood counts, liver function tests, coagulation factors, and zinc and ammonia levels. The Child-Turcotte-Pugh (CTP) and Model for End-stage Liver Disease (MELD) calculated scores were evaluated. Patient follow-up was every four weeks for medical adherence and patients were monitored for adverse events. Extra visits were allowed in the case of any adverse events. At the end of the 12-week treatment period, each patient repeated the NP tests and SF-36 questionnaire. Blood samples for biochemical indexes, including serum ammonia, were also taken at 12 weeks in both groups.

\section{Outcomes}

The primary outcome was the absolute mean change of NP tests from baseline and after 12 weeks of zinc supplementation compared with placebo. Secondary outcomes were the mean change in HRQOL, serum zinc and ammonia levels, aminotransferases, albumin, platelet count and INR after treatment compared with baseline. When comparing subgroups divided by baseline zinc level, we compared the effect of zinc supplementation on psychomotor tests and quality of life. The safety of zinc was monitored in terms of adverse events. Adverse events were assessed using the Common Terminology Criteria for Adverse Events (version 5.0).

\section{Statistical analysis}

All continuous variables are expressed as mean \pm standard deviation (SD), whereas categorical variables are expressed as absolute values and percentages. To compare between groups, continuous variables were analyzed by the Independent $t$ test and the WilcoxonMann-Whitney test. To compare the mean change $(95 \% \mathrm{CI})$ between groups in the subgroup analysis, we used the Independent $t$ test or Mann-Whitney test. Data were analyzed by SPSS version 22 and statistical significance was determined at $\mathrm{P}<0.05$.

Ethical approval was obtained by the Human Research Ethics Committee of Vajira Hospital, Thailand, and the study was conducted according to the good clinical practice guidelines, as well as the Declaration of Helsinki. All data were fully anonymized before they were assessed. The project number for ethical approval was 001-2020. This study was registered in Thai clinical trial registration No 202104233001.

\section{Sample Size}

As Mousa (2016) reported in his previous work, the mean and SD of the NCT-A and DST between the intervention group (antioxidants/zinc) and the control group (lactulose) after three months of the treatment; for NCT-A, the time at the end of treatment was $56.8 \pm 3.07$ and $64.3 \pm 5.31 \mathrm{sec}$, respectively $(\mathrm{P}<0.001)$, and for DST, the points at the end of treatment were $12.36 \pm 0.87$ and $11.58 \pm 1.19$, respectively $(\mathrm{P}<0.006)$. The minimum sample size after considering the $95 \% \mathrm{CI}$ and $80 \%$ power of the test of this study will be 29 in each group. After adding a $10 \%$ attrition rate, at least 33 subjects in each group will participate in the study.

\section{Results}

A total of 157 cirrhotic patients were screened from January to December 2020. Thirty-two patients met the exclusion criteria and were therefore ineligible, while two others declined to participate. One hundred and twentyfive patients underwent NP tests, of whom 69 (55\%) patients were diagnosed with MHE and were randomly assigned into two groups: one group (35 patients) received elemental zinc three times a day with a total dose of 45 mg per day for 12 weeks, and the other (34 patients) received placebo for the same duration. After 12 weeks, we repeated the laboratory measurements, NP test and SF-36 questionnaire. During our follow-up visits, three patients were removed from the study due to the COVID-19 pandemic.

In total, $69 \mathrm{MHE}$ patients were enrolled in the study with a mean age of $58.9 \pm 8.6$ years; fifty-six patients $(81 \%)$ were male. The etiology of cirrhosis was alcohol (34.8\%; $\mathrm{n}=24)$ followed by chronic hepatitis C (33.3\%; $\mathrm{n}=23)$, chronic hepatitis B $(18.8 \% ; \mathrm{n}=13)$, non-alcoholic steatohepatitis $(8.7 \% ; n=6)$ and a small number of cases of autoimmune liver disease $(4.3 \%$; $n=3)$. Most patients had a compensated Child-Pugh A without ascites. Just over a quarter $(27 \%)$ of the patients were currently receiving lactulose treatment to prevent clinical hepatic encephalopathy. In patients $(n=19)$ who used lactulose for HE prophylaxis, baseline ammonia levels did not differ from the level in non-lactulose therapy $(97.7 \pm 55.7$ vs. $90.5 \pm 61.1 \mu \mathrm{mol} / \mathrm{L}, \mathrm{P}=0.65)$. None of the patients in our study had a history of previous overt hepatic

Table 1. Baseline Characteristics of Cirrhotic Patients with Minimal Hepatic Encephalopathy $(n=69)$

\begin{tabular}{|c|c|c|c|}
\hline & $\begin{array}{l}\text { Zinc group } \\
(\mathrm{n}=35)\end{array}$ & $\begin{array}{c}\text { Placebo } \\
\text { group }(n=34)\end{array}$ & p-value \\
\hline \multicolumn{4}{|l|}{ Sex } \\
\hline Female & $6(17.1 \%)$ & $7(20.6 \%)$ & 0.77 \\
\hline Male & $29(82.9 \%)$ & $27(79.4 \%)$ & 0.77 \\
\hline Age & $60.06 \pm 8.1$ & $57.74 \pm 9.1$ & 0.27 \\
\hline BMI, $\mathrm{kg} / \mathrm{m}^{2}$ & $24.96 \pm 4.8$ & $24.9 \pm 4.8$ & 0.96 \\
\hline Education, years & $7.09 \pm 3.4$ & $8.79 \pm 4.1$ & 0.07 \\
\hline \multicolumn{4}{|l|}{ Etiology of cirrhosis; $\mathrm{n}(\%)$} \\
\hline Chronic hepatitis $\mathrm{C}$ & $15(42.9 \%)$ & $8(23.5 \%)$ & 0.13 \\
\hline Alcohol & $11(31.4 \%)$ & $13(38.2 \%)$ & 0.62 \\
\hline Chronic hepatitis B & $6(17.1 \%)$ & $7(20.6 \%)$ & 0.77 \\
\hline Non-alcoholic steatohepatitis & $3(8.6 \%)$ & $3(8.8 \%)$ & 1 \\
\hline Autoimmune hepatitis & $0(0 \%)$ & $2(5.9 \%)$ & 0.24 \\
\hline Primary biliary cholangitis & $0(0 \%)$ & $1(2.9 \%)$ & 0.5 \\
\hline Present of esophageal varices & $9(25.7 \%)$ & $4(11.8 \%)$ & 0.22 \\
\hline Ascites & $1(2.9 \%)$ & $1(2.9 \%)$ & 1 \\
\hline $\begin{array}{l}\text { Current treatment with } \\
\text { beta-blocker }\end{array}$ & $8(22.9 \%)$ & $2(5.9 \%)$ & 0.08 \\
\hline $\begin{array}{l}\text { Current treatment with } \\
\text { lactulose }\end{array}$ & $10(28.6 \%)$ & $9(26.5 \%)$ & 1 \\
\hline \multicolumn{4}{|l|}{ Child-Pugh score } \\
\hline A & $33(94.3 \%)$ & $31(91.2 \%)$ & 0.673 \\
\hline B & $2(5.7 \%)$ & $3(8.8 \%)$ & 0.673 \\
\hline
\end{tabular}

Value presented as mean \pm SD. and $\mathrm{n}(\%)$. P-value corresponds to Independent $t$ and Fisher's exact test.

Asian Pacific Journal of Cancer Prevention, Vol 22 
Table 2. Baseline Results of Neuropsychometric Test, SF-36 Scores and Laboratory Parameters

\begin{tabular}{lccc}
\hline & $\begin{array}{c}\text { Zinc group } \\
(\mathrm{n}=35) \\
\text { mean } \pm \mathrm{SD}\end{array}$ & $\begin{array}{c}\text { Placebo group } \\
(\mathrm{n}=34)\end{array}$ & p-value \\
mean $\pm \mathrm{SD}$ & \\
\hline NCT-A, sec & $64.71 \pm 19.2$ & $71.5 \pm 43.3$ & 0.41 \\
NCT-B, sec & $152.91 \pm 44.7$ & $136.68 \pm 53.0$ & 0.17 \\
SDT, sec & $113.97 \pm 30.5$ & $105.12 \pm 26.6$ & 0.2 \\
LTT, sec & $190.6 \pm 44.2$ & $168.12 \pm 47.6$ & 0.046 \\
DST, point & $18.74 \pm 6.4$ & $20.82 \pm 8.8$ & 0.27 \\
PF & $65.86 \pm 12.5$ & $65.56 \pm 16.9$ & 0.93 \\
SF & $77.63 \pm 18.8$ & $78.88 \pm 20.9$ & 0.79 \\
MH & $65.6 \pm 16.2$ & $69.53 \pm 17.8$ & 0.34 \\
BP & $59.83 \pm 18.8$ & $63.85 \pm 26.8$ & 0.47 \\
PH & $52.43 \pm 20.1$ & $51.62 \pm 18.4$ & 0.86 \\
RP & $57.86 \pm 40.1$ & $54.41 \pm 42.4$ & 0.73 \\
RM & $52.17 \pm 37.5$ & $52.94 \pm 45.1$ & 0.94 \\
VT & $57.94 \pm 16.2$ & $58.24 \pm 18.9$ & 0.95 \\
SF-36 & $489.31 \pm 125.4$ & $495.03 \pm 160.1$ & 0.87 \\
ALT, U/L & $44.51 \pm 28.1$ & $56.03 \pm 35.9$ & 0.14 \\
Albumin, g/dL & $4.03 \pm 0.5$ & $4.03 \pm 0.6$ & 0.97 \\
Platelets x 10 & $161.4 \pm 78.3$ & $145.1 \pm 71.9$ & 0.37 \\
INR & $1.1 \pm 0.1$ & $1.18 \pm 0.2$ & 0.06 \\
Zinc, mcg/dL & $68.47 \pm 20.6$ & $68.65 \pm 16.7$ & 0.97 \\
Ammonia, $\mu$ mol/L & $89.1 \pm 61.2$ & $95.99 \pm 58.1$ & 0.63 \\
MELD & $9 \pm 3.1$ & $9.15 \pm 2.5$ & 0.83 \\
CTP & $5.17 \pm 0.5$ & $5.32 \pm 0.6$ & 0.28 \\
\hline Vale pro & & & \\
& & &
\end{tabular}

Value presented as mean \pm SD and $\mathrm{n}(\%)$ in NP test and laboratory. And presented as median (IQR) in SF-36 test. P-value corresponds to Independent $t$ and Fisher's exact test. NCT-A, number connection test part A; NCT-B, number connection test part B; DST, digit symbol test; SDT, serial dotting test; LTT, line tracing test; MELD, Model for End-Stage Liver Disease; CTP, Child-Turcotte-Pugh; PF, physical functioning; SF, social functioning; $\mathrm{MH}$, mental health; $\mathrm{BP}$, bodily pain; $\mathrm{PH}$, perception in health; RP, role-physical; RE, role emotional; VT, vitality

encephalopathy.

There was no difference in the results for NCT-A, NCT-B, SDT and DST tests between the two groups. At baseline, there was a longer delay time for line tracing test in the zinc supplement group (190.6 \pm 44.2 vs. $168.12 \pm 47.6 \mathrm{sec}, \mathrm{p}=0.046)$. Baseline serum zinc in our MHE patients showed a marginal deficiency $(68.6 \pm 18.6$ $\mathrm{mcg} / \mathrm{dL}$ ) and baseline serum ammonia ranged from 29.1 to $368.8 \mu \mathrm{mol} / \mathrm{L}$, without any significant difference between the two groups. Neither baseline synthetic function nor baseline SF-36 scores were different between the two groups ( $p=0.97$ and $p=0.87$, respectively) as shown in Table 2 .

\section{Effect of zinc supplementation on NP tests}

After 12 weeks of zinc supplementation, there was an improvement in the performance of NCT-A, NCT-B, and LTT, and there was a statistically significant improvement in SDT $(113.97 \pm 30.5$ vs. $108.32 \pm 29.6$; $\mathrm{p}=0.003)$ and DST from baseline (18.74 \pm 6.4 vs. 20.5 $\pm 6.3 ; \mathrm{p}=0.011)$. In comparison with placebo, the mean change in improvement in most tests was significantly for zinc therapy (NCT-A: $p=0.029$; NCT-B: $p=0.008$, SDT: $p=0.002$; DST: $p<0.001)$. The mean change in reduction of time of LTT after therapy was not significant in comparison between both groups. (Table 3)

Effect of zinc supplementation on laboratory parameters

After zinc supplementation, serum zinc increased from 68.47 to $91.21 \mathrm{mcg} / \mathrm{dL}$. Even though we observed that either an increased platelet count, an improvement in INR or an increase in serum albumin were established from baseline compared with 12 weeks in the zinc therapy group ( $\mathrm{p}=0.037, \mathrm{p}=0.045, \mathrm{p}=0.041$; respectively), the mean changes in these parameters were not significant when compared with the mean changes in the placebo group. After twelve weeks of zinc therapy, there was a trend of declining serum ammonia $(89.1 \pm 61.2$ vs. $72.7 \pm 27.5 ; \mathrm{p}=0.058$ ). Neither CTP nor MELD showed an improvement after treatment in both groups (Table 3 ).

\section{Effect of zinc supplementation on HRQOL}

In terms of the quality of life questionnaire survey, there was a significant improvement in SF-36 score $(p<0.001)$ including each aspect in terms of physical function, social function, mental health, body pain, physical health, role limitations due to emotional problems and vitality after zinc therapy $(\mathrm{p}=0.004, \mathrm{p}<0.001, \mathrm{p}=$ $0.041, \mathrm{p}=0.002, \mathrm{p}=0.001, \mathrm{p}=0.002$, and $\mathrm{p}=0.021$, respectively). In comparison with placebo, a significant improvement in the mean SF-36 score was seen with zinc supplementation in most domains, with the exceptions of bodily pain and vitality (Table 3 ).

As the definition of zinc deficiency was a level lower than $60 \mathrm{mcg} / \mathrm{dL}$ (Yanagisawa, 2008; Somi et al., 2012), 12 of the $35(34.3 \%)$ patients had baseline zinc deficiencies. In the baseline zinc deficiency group, the ammonia level was no different compared with the other groups $(105.3+92$ vs. $80.9+41 \mu \mathrm{mol} / \mathrm{L}, \mathrm{p}=0.29)$. According to comparisons between subgroups separated by baseline zinc level, after 12 weeks of zinc therapy, there was no difference in mean change of improvement in NP test or mean change in improvement of SF-36 score, together with nearly all domains of the questionnaire, except for a higher mean change in mental health which was observed in the baseline normal zinc level.

There was no significant difference reported in side effect events in both groups. Three of the $66(4.5 \%)$ patients reported minor adverse events. The adverse events in both groups were nausea in 1 patient $(2.9 \%)$ in the zinc group and in 1 patient $(3.1 \%)$ in the placebo group. Symptoms of dizziness were reported in only 1 patient in the zinc therapy group. Dizziness and nausea in our study were clarified as grade 1 severity by definition of Common Terminology Criteria for Adverse Events; all 3 patients were self-limited.

\section{Discussion}

A diagnosis of MHE has often been overlooked by real world practice. MHE is a condition which has a significant 


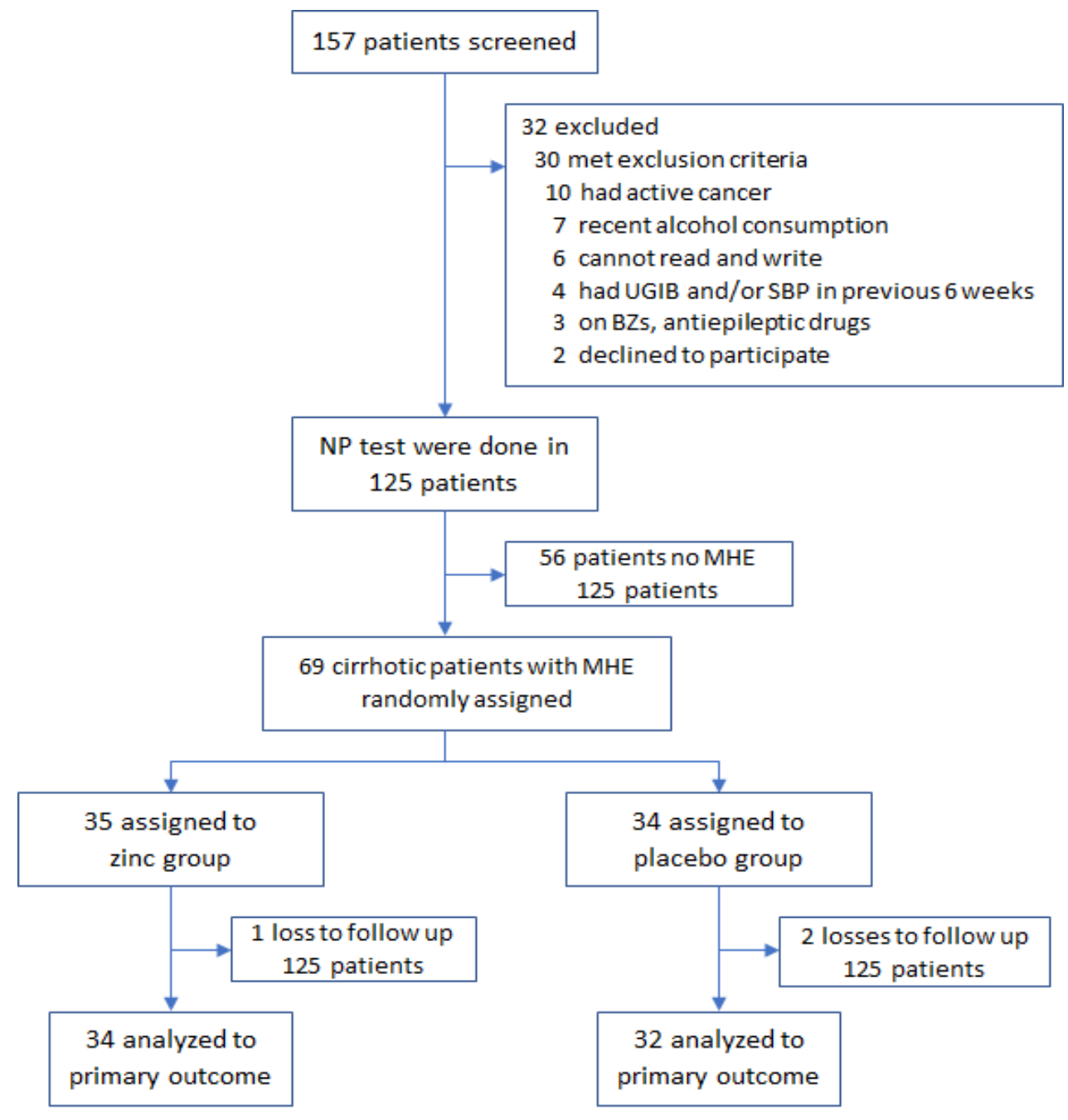

Figure 1. Study Profile

detrimental impact on daily quality of life in patients with cirrhosis. The previous prevalence of MHE in cirrhotic patients ranges from 40-60\% (Maldonado-Garza et al., 2011; Rathi et al., 2019; Elgohary et al., 2020; Das et al., 2001). In our study, the prevalence of MHE was 55\% in compensated cirrhotic patients.

To the best of our knowledge, zinc deficiency is common in chronic liver disease patients, especially in cirrhosis. Zinc deficiency occurred as the result of several mechanisms such as poor dietary intake, diminished absorption, decreased metabolism, reduction capacity to bind albumin and enhanced protein catabolism (Himoto and Masaki, 2018; Grüngreiff et al., 2016; Kamani and Shaikh, 2018). Zinc deficiency is also a precipitating factor for both covert and overt HE. The prevalence of zinc deficiency is approximately $69 \%$ in patients with advanced cirrhosis but the prevalence of this deficiency was not correlated with grading of HE (Loomba et al., 1995; Katayama et al., 2018). The mean zinc level in our MHE patients was $68.6 \pm 18.6 \mathrm{mcg} / \mathrm{dL}$, which indicates a marginal level of deficiency. Even though the mean zinc level in our study was higher than the level in previous MHE research, nearly one-third of our patients (21 of 69; $30.4 \%$ ) had baseline zinc deficiency (Mousa et al., 2016).

The positive impact of zinc supplement as an add-on standard therapy in cirrhotic patients with hepatic encephalopathy was reported in several studies, and can improve the performance of many psychomotor tests, especially the number connection test (Mousa et al., 2016; Takuma et al., 2010; Bresci et al., 1993). Our prospective, randomized, controlled trial study conducted by the use of psychometric hepatic encephalopathy score (PHES) comprised 5 sub-tests, four of which were simple (NCT-A, NCT-B, SDT and DST) and which tested psychomotor speed, attention, and set shifting. The other sub-test tool was assessed for both psychomotor accuracy and additional visuomotor ability examination (LTT). LTT is a test that evaluates a combination of visual performance with movement skills to produce actions. Our results showed a beneficial effect of 12-week zinc monotherapy, as well as a significant improvement in NCT-A, NCT-B, SDT and DST and a trend towards the shortened time for LTT, which currently limits the evaluated by other studies.

As in aspect of quality of life, previously reported HRQOL score survey in MHE was significantly lower than the HRQOL score in cirrhosis without MHE. A survey revealed that the mean SF-36 score in our MHE patients was much higher than in a previous Chinese Survey in cirrhosis Child A without any grade of encephalopathy (Bo et al., 2007). However, we found an impairment in daily function in our MHE patients following assessment of their HRQOL with the SF-36 general questionnaire. This 


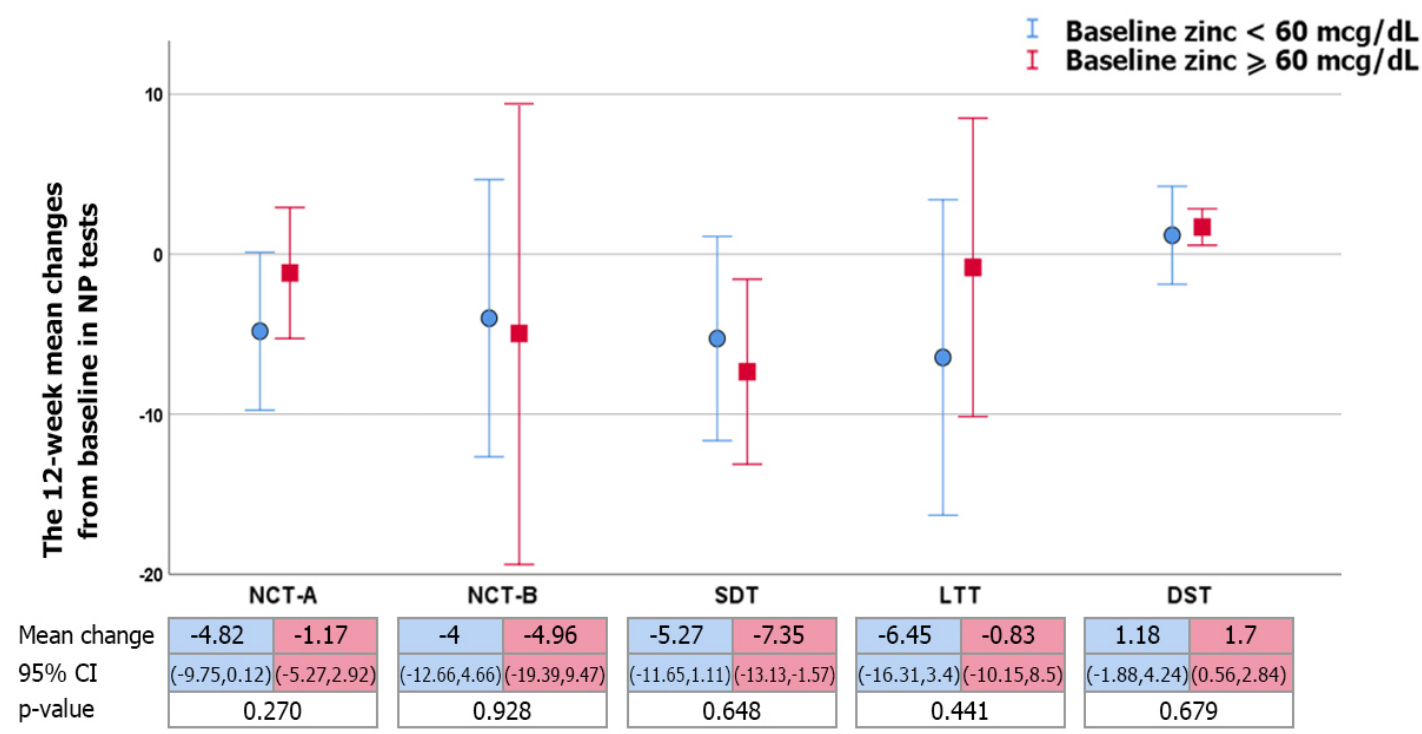

Figure 2A. The 12-Week Mean Changes (95\%CI) of Neuropsychometric Tests in Zinc Supplement Group, Compared between Baseline Zinc Level $<60 \mathrm{mcg} / \mathrm{dL}$ (blue line) and Zinc Level $\geq 60 \mathrm{mcg} / \mathrm{dL}$ (red line) Group, as Determined via Independent $t$ Test. Abbreviations: NCT-A, number connection test-A; NCT-B, number connection test-B, SDT, serial dot test; LTT, line tracing test; DST, digit symbol test

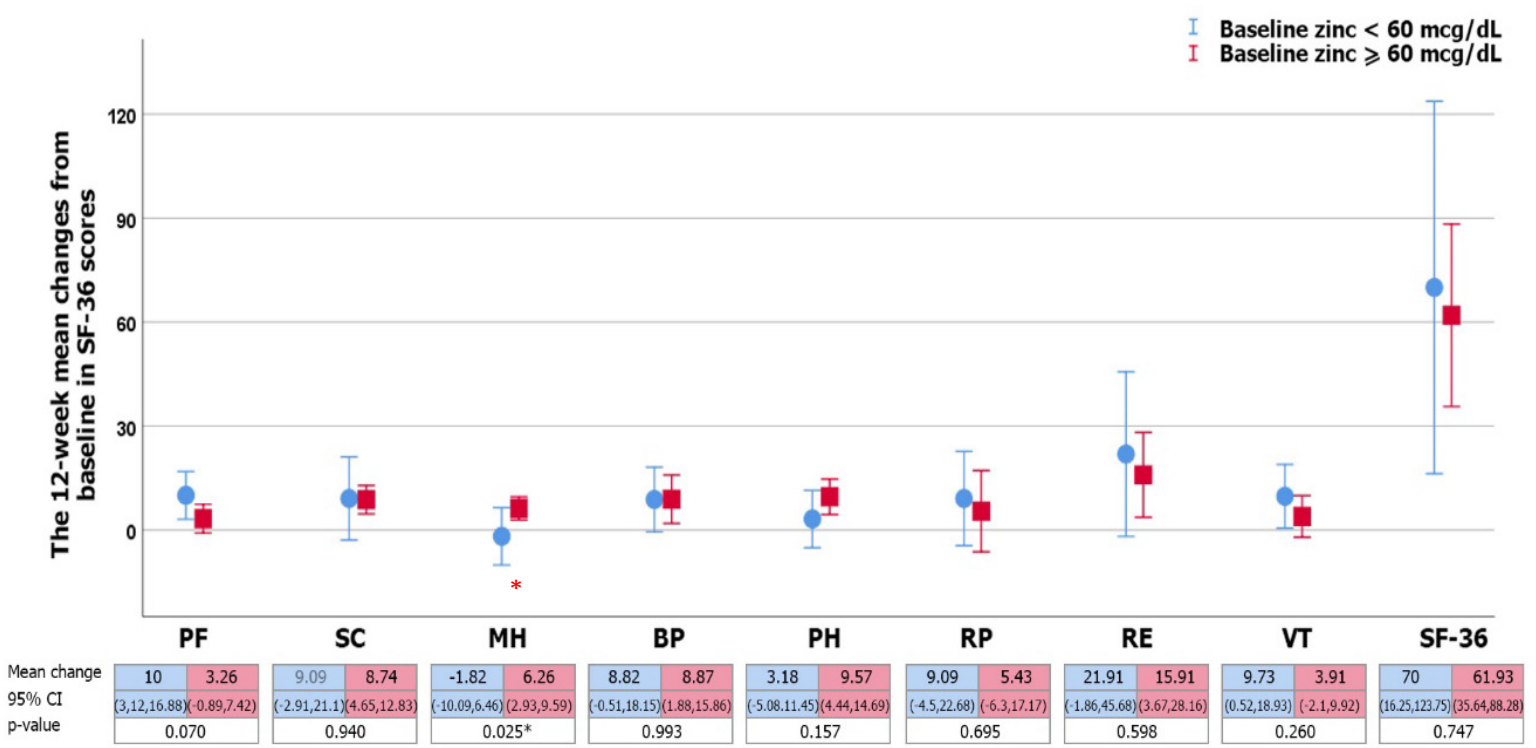

Figure 2B. The 12-Week Mean Changes (95\%CI) of SF-36 Tests in Zinc Supplement Group, Compared between Baseline Zinc Level $<60 \mathrm{mcg} / \mathrm{dL}$ (blue line) and Zinc Level $\geq 60 \mathrm{mcg} / \mathrm{dL}$ (red line) Group. *Statistically significant (p $<0.05)$ comparison between groups as determined via the Independent $t$ test. Abbreviations: PF, physical functioning; $\mathrm{SF}$, social functioning; $\mathrm{MH}$, mental health; $\mathrm{BP}$, bodily pain; $\mathrm{PH}$, perception in health; RP, role-physical; RE, role emotional; VT, vitality

Figure 2. Change of Mean (95\% CI) Neuropsychometric Tests and Change of Mean SF-36 Scores According to Baseline Zinc Level in Zinc Supplement Group

was a $20 \%$ decrease in the score compared with healthy populations (Blake et al., 2000).

Zinc supplementation has a benefit not only by improving of attention but also in cognitive function, including memory. Our HRQOL survey based on the total SF-36 score was significantly improved after zinc monotherapy. When compared with placebo, at least 5 important domains in physical function, social function, perception of health, role emotional and mental health were significantly improved after zinc supplementation.
Even though there was an increase in the mean change in bodily pain, limited physical role and vitality in the zinc group, this change was not different in comparison with the other group.

Zinc is a cofactor of ornithine transcarbamylase, which promotes liver ammonia detoxification and the elimination of ammonia from skeletal muscle via the activation of glutamine synthetase. We found baseline serum ammonia to show wide-ranging levels in our MHE patients with a maximal value that was 7-times higher than the upper 
Table 3. Neuropsychometric Tests, SF-36 Scores and Laboratory Pparameters at Baseline and after 12-week of Therapy in Both Groups

\begin{tabular}{|c|c|c|c|c|c|c|c|}
\hline & \multicolumn{3}{|c|}{ Zinc Group } & \multicolumn{3}{|c|}{ Placebo Group } & \multirow[t]{2}{*}{ P3 } \\
\hline & $\begin{array}{c}\text { Baseline } \\
\text { mean } \pm \mathrm{SD}\end{array}$ & $\begin{array}{c}12 \text { Weeks } \\
\text { mean } \pm \text { SD }\end{array}$ & P1 & $\begin{array}{c}\text { Baseline } \\
\text { mean } \pm \text { SD }\end{array}$ & $\begin{array}{c}12 \text { Weeks } \\
\text { mean } \pm \text { SD }\end{array}$ & P2 & \\
\hline NCT-A, sec & $64.71 \pm 19.2$ & $62.76 \pm 17.3$ & 0.132 & $71.5 \pm 43.3$ & $75.38 \pm 42.4$ & 0.113 & $0.029^{*}$ \\
\hline NCT-B, sec & $152.91 \pm 44.7$ & $148.53 \pm 38.8$ & 0.343 & $136.68 \pm 53.0$ & $150.87 \pm 51.7$ & $<0.001 *$ & $0.008^{*}$ \\
\hline SDT, sec & $113.97 \pm 30.5$ & $108.32 \pm 29.6$ & $0.003 *$ & $105.12 \pm 26.6$ & $110.41 \pm 29.8$ & 0.104 & $0.002 *$ \\
\hline LTT, sec & $190.6 \pm 44.2$ & $188.24 \pm 41.4$ & 0.436 & $168.12 \pm 47.6$ & $164.63 \pm 39.4$ & 0.154 & 0.855 \\
\hline DST, sec & $18.74 \pm 6.4$ & $20.5 \pm 6.3$ & $0.011 *$ & $20.82 \pm 8.8$ & $19.09 \pm 7.4$ & 0.011 & $<0.001 *$ \\
\hline $\mathrm{PF}$ & $65.86 \pm 12.5$ & $71.62 \pm 9.9$ & $0.004 *$ & $65.56 \pm 16.9$ & $63.19 \pm 18.5$ & 0.515 & $0.013^{*}$ \\
\hline SF & $77.63 \pm 18.8$ & $86.79 \pm 16.3$ & $<0.001 *$ & $78.88 \pm 20.9$ & $79.28 \pm 23.4$ & 0.669 & $0.017 *$ \\
\hline $\mathrm{MH}$ & $65.6 \pm 16.2$ & $68.94 \pm 12.2$ & 0.041 & $69.53 \pm 17.8$ & $67 \pm 17.8$ & 0.154 & $0.014 *$ \\
\hline $\mathrm{BP}$ & $59.83 \pm 18.8$ & $68.79 \pm 16.4$ & 0.002 & $63.85 \pm 26.8$ & $65.72 \pm 22.3$ & 0.319 & 0.074 \\
\hline $\mathrm{PH}$ & $52.43 \pm 20.1$ & $59.71 \pm 18.1$ & 0.001 & $51.62 \pm 18.4$ & $50 \pm 20.8$ & 0.709 & $0.018^{*}$ \\
\hline $\mathrm{RP}$ & $57.86 \pm 40.1$ & $63.97 \pm 37$ & 0.13 & $54.41 \pm 42.4$ & $50.78 \pm 40.4$ & 0.374 & 0.096 \\
\hline $\mathrm{RM}$ & $52.17 \pm 37.5$ & $70.59 \pm 36.5$ & 0.002 & $52.94 \pm 45.1$ & $59.41 \pm 41.3$ & 0.13 & $0.047^{*}$ \\
\hline VT & $57.94 \pm 16.2$ & $63.09 \pm 13.2$ & 0.021 & $58.24 \pm 18.9$ & $58.75 \pm 19.3$ & 0.802 & 0.085 \\
\hline SF-36 & $489.31 \pm 125.4$ & $553.5 \pm 110.4$ & $<0.001 *$ & $495.03 \pm 160.1$ & $494.13 \pm 165.9$ & 0.98 & $<0.001 *$ \\
\hline ALT, U/L & $44.51 \pm 28.1$ & $39.85 \pm 25.9$ & 0.103 & $56.03 \pm 35.9$ & $57.06 \pm 40.2$ & 0.954 & 0.489 \\
\hline Albumin, $\mathrm{g} / \mathrm{dL}$ & $4.03 \pm 0.53$ & $4.2 \pm 0.47$ & $0.041 *$ & $4.03 \pm 0.58$ & $4.23 \pm 0.53$ & $0.004 *$ & 0.433 \\
\hline Platelets $\times 10^{3}$ & $161.4 \pm 78.3$ & $171.6 \pm 84.8$ & $0.037 *$ & $145.1 \pm 71.9$ & $147.6 \pm 73.3$ & 0.904 & 0.089 \\
\hline PT, sec & $13.23 \pm 1.2$ & $13.21 \pm 1.2$ & 0.98 & $13.73 \pm 1.7$ & $13.65 \pm 1.7$ & 0.267 & 0.482 \\
\hline INR & $1.1 \pm 0.1$ & $1.12 \pm 0.1$ & $0.045^{*}$ & $1.18 \pm 0.2$ & $1.16 \pm 0.1$ & 0.354 & 0.136 \\
\hline Zinc, $\mathrm{mcg} / \mathrm{dL}$ & $68.47 \pm 20.6$ & $91.21 \pm 28.5$ & $<0.001^{*}$ & $68.65 \pm 16.6$ & $66.25 \pm 15.2$ & 0.42 & $<0.001 *$ \\
\hline Ammonia, $\mu \mathrm{mol} / \mathrm{L}$ & $89.1 \pm 61.2$ & $72.71 \pm 27.5$ & 0.058 & $95.99 \pm 58.1$ & $85.54 \pm 44.0$ & 0.129 & 0.058 \\
\hline CTP score & $5.17 \pm 0.5$ & $5.03 \pm 0.1$ & 0.058 & $5.32 \pm 0.6$ & $5.19 \pm 0.4$ & 0.057 & 0.93 \\
\hline MELD & $9 \pm 3.06$ & $8.74 \pm 1.7$ & 0.561 & $9.15 \pm 2.5$ & $9.5 \pm 2.7$ & 0.36 & 0.288 \\
\hline
\end{tabular}

Value presented as mean \pm SD and $\mathrm{n}(\%)$ in NP test and laboratory. And presented as median (IQR) in SF-36 test. P-value corresponds to Independent t and Fisher's exact test. NCT-A, number connection test part A; NCT-B, number connection test part B; DST, digit symbol test; SDT, serial dotting test; LTT, line tracing test; MELD, Model for End-Stage Liver Disease; CTP, Child-Turcotte-Pugh; PF, physical functioning; SF, social functioning; $\mathrm{MH}$, mental health; BP, bodily pain; PH, perception in health; RP, role-physical; RE, role emotional; VT, vitality; P1, Mean change before and after zinc therapy; P2, Mean change before and after placebo; P3, Mean change after therapy compared between group

normal limit. After zinc therapy, blood ammonia levels decreased by approximately $20 \%$; however, there was only a trend towards the non-significant reduction in ammonia levels after zinc supplementation. That was why it was not possible to conclude that the better performance in NP test after zinc Px was caused by a decrease in serum ammonia.

There are many studies of changes in serum ammonia after zinc Px which give conflicting results. Previous studies have shown that zinc supplementation can result in lower ammonia levels in HE grade 1-2 but not in the reduction of recurrent HE (Blake et al., 2000; Riggio et al., 1991; Chavez-Tapia et al., 2013). There was a previous RCT trial of a small number of cirrhotic patients, with varying degrees of $\mathrm{HE}$ with hyperammonemia, who were given zinc acetate supplementation at a dosage of $150 \mathrm{mg}$ / day (elemental zinc $45 \mathrm{mg} /$ day) for 3 months, the results of which showed a reduction of serum ammonia but the sample population was too small to clarify a benefit of lowering serum ammonia in the condition of MHE (Katayama et al., 2014). The positive impact of zinc on ammonia level was supported by Hayashi et al., who reported the combination of zinc sulfate $200-600 \mathrm{mg} /$ day (elemental zinc 46-138 mg/day) with branch chain amino acids for 6 months reduced serum ammonia significantly without mentioning the stage of HE (Hayashi et al., 2007).

Most previous HE trials were designed to study the benefit of additional zinc supplementation with lactulose on either serum ammonia, psychomotor test performance or HRQOL (Shiha and Mousa, 2019; Mousa et al., 2016; Takuma et al., 2010; Bresci et al., 1993). On the other hand, in Mousa (2016) studied RCT trials, in particular MHE stage, and reported that three month combination of zinc gluconate $175 \mathrm{mg}$ /day (elemental zinc $25 \mathrm{mg} /$ day) plus antioxidant and lactulose in comparison with only lactulose, which resulted in zinc supplementation with the antioxidant group, can improve NCT-A but produced no difference in the reduction of serum ammonia levels when compared with lactulose therapy alone. Recently, a metaanalysis of the addition of zinc with lactulose in cirrhosis with mild $\mathrm{HE}$ (< grade 2) for 3-6 months demonstrated improved NCT tests but did not significantly lower serum ammonia levels when compared with lactulose monotherapy (Shiha and Mousa, 2019). Our study focused on MHE stage and emphasized a positive trend of 
ammonia reduction together with an improvement in both PHES and HRQOL after 3 months of zinc monotherapy irrespective of the baseline zinc level. Furthermore, whether the continuation of zinc supplementation for longer periods or dose modification may impact serum ammonia and PHES scores in terms of LTT may be of interest in future research.

We observed minor side effects after zinc supplementation in only 2 patients and their symptoms occurred for a few days and self-limited with continuing therapy. The reason why patients maintained good drug compliance was because the form of oral zinc supplement in our study was zinc amino acid (bisglycinate) chelate. It was absorbed differently from other forms of zinc (zinc sulfate, zinc oxide). This zinc bisglycinate consists of one zinc molecule bound to two small molecules of the amino acid glycine. This form was stable in acidic conditions and can be absorbed without decomposition into cationic zinc. In addition, zinc bisglycinate was safer and welltolerated, with less nausea and vomiting, compared with other zinc formulations (Gandia et al., 2017; DiSilvestro and Swan, 2008). Our study needs a higher dosage and longer duration of zinc supplementation.

In conclusion, the zinc supplementation in cirrhotic patients with MHE for at least 12 weeks could demonstrate positive effects on psychomotor performance and improved HRQOL irrespective of baseline zinc levels.

\section{Author Contribution Statement}

Rattaya Janyajirawong and Supatsri Sethasine contributed to the study design. Rattaya Janyajirawong contributed to conduct protocol and data collection. Supatsri Sethasine contributed to data analysis and manuscript writing. Ratha-korn Vilaichone contributed to intensive review of the manuscript. All authors reviewed the final results and approved the final version of the manuscript.

\section{Acknowledgements}

This study was approved by the ethics committee of Vajira hospital, Navamindradhiraj University, Bangkok, Thailand (COA No.001-2020).

\section{Funding Statement}

This study was supported by a grant from Navamindradhiraj University Research Fund and the Gastroenterological Association of Thailand.

\section{Conflict of Interest Statement}

None of the authors have a conflict of interest.

\section{References}

Agrawal S, Umapathy S, Dhiman RK (2015). Minimal hepatic encephalopathy impairs quality of life. J Clin Exp Hepatol, 5, 42-8.

Ampuero J, Montoliú C, Simón-Talero M, et al (2018). Minimal hepatic encephalopathy identifies patients at risk of faster cirrhosis progression. J Gastroenterol Hepatol, 33, 718-25.
Bajaj JS, Duarte-Rojo A, Xie JJ, et al (2020). Minimal hepatic encephalopathy and mild cognitive impairment worsen quality of life in elderly patients with cirrhosis. Clin Gastroenterol Hepatol, 18, 3008-16.e2.

Bajaj JS, Saeian K, Hafeezullah M, et al (2008). Patients with minimal hepatic encephalopathy have poor insight into their driving skills. Clin Gastroenterol Hepatol, 6, 1135-9.

Blake C, Codd MB, O'Meara YM (2000). The Short Form 36 (SF-36) Health Survey: normative data for the Irish population. Ir J Med Sci, 169, 195-200.

Bo ZJ, Qiu DK, Ma X, et al (2007). Assessment of the health-related quality of life of patients with minimal hepatic encephalopathy. Zhonghua Gan Zang Bing Za Zhi, 15, 412-6.

Bresci G, Parisi G, Banti S (1993). Management of hepatic encephalopathy with oral zinc supplementation: a long-term treatment. Eur J Med, 2, 414-6.

Chavez-Tapia NC, Cesar-Arce A, Barrientos-Gutiérrez T, et al (2013). A systematic review and meta-analysis of the use of oral zinc in the treatment of hepatic encephalopathy. Nutr J, 12, 74.

Ciećko-Michalska I, Szczepanek M, Słowik A, et al (2012). Pathogenesis of hepatic encephalopathy. Gastroenterol Res Pract, 2012, 642108.

Das A, Dhiman RK, Saraswat VA, et al (2001). Prevalence and natural history of subclinical hepatic encephalopathy in cirrhosis. J Gastroenterol Hepatol, 16, 531-5.

DiSilvestro RA, Swan M (2008). Comparison of four commercially available zinc supplements for performance in a zinc tolerance test. Faseb J, 22, 93.3-693.3.

Elgohary MN, Amer K, Bassiony M (2020). Prevalence and risk factors of minimal hepatic encephalopathy in patients with compensated liver cirrhosis. Egypt J Hosp Med, 80, 871-5.

Felipo V, Ordoño JF, Urios A, et al (2012). Patients with minimal hepatic encephalopathy show impaired mismatch negativity correlating with reduced performance in attention tests. Hepatology, 55, 530-9.

Gandia P, Bour D, Maurette JM, et al (2017). A bioavailability study comparing two oral formulations containing zinc ( $\mathrm{Zn}$ bis-glycinate vs. Zn gluconate) after a single administration to twelve healthy female volunteers. Int J Vitam Nutr Res, 77, 243-8.

García-García R, Cruz-Gómez Á J, Urios A, et al(2018). Learning and memory impairments in patients with minimal hepatic encephalopathy are associated with structural and functional connectivity alterations in hippocampus. Sci Rep, 8, 9664.

Grüngreiff K, Reinhold D, Wedemeyer H (2016). The role of zinc in liver cirrhosis. Ann Hepatol, 15, 7-16.

Hayashi M, Ikezawa K, Ono A, et al (2007). Evaluation of the effects of combination therapy with branched-chain amino acid and zinc supplements on nitrogen metabolism in liver cirrhosis. Hepatol Res, 37, 615-9.

Himoto T, Masaki T (2018). Associations between zinc deficiency and metabolic abnormalities in patients with chronic liver disease. Nutrients, 10, 88.

Kamani L, Shaikh H (2018). Zinc level assessment in patients having viral cirrhosis. PakJ Med Sci, 34, 478-81.

Katayama K (2020). Zinc and protein metabolism in chronic liver diseases. Nutr Res, 74, 1-9.

Katayama K, Kawaguchi T, Shiraishi K, et al (2018). The prevalence and implication of zinc deficiency in patients with chronic liver disease. J Clin Med Res, 10, 437-44.

Katayama K, Saito M, Kawaguchi T, et al (2014). Effect of zinc on liver cirrhosis with hyperammonemia: a preliminary randomized, placebo-controlled double-blind trial. Nutrition, 30, 1409-14.

Li SW, Wang K, Yu YQ, et al (2013). Psychometric hepatic 
encephalopathy score for diagnosis of minimal hepatic encephalopathy in China. World $J$ Gastroenterol, 19, 8745-51.

Loomba V, Pawar G, Dhar KL, et al (1995). Serum zinc levels in hepatic encephalopathy. Indian J Gastroenterol, 14, 51-3.

Maldonado-Garza HJ, Vázquez-Elizondo G, Gaytán-Torres JO, et al (2011). Prevalence of minimal hepatic encephalopathy in cirrhotic patients. Ann Hepatol, 10, 40-4.

Marchesini G, Fabbri A, Bianchi G, et al (1996). Zinc supplementation and amino acid-nitrogen metabolism in patients with advanced cirrhosis. Hepatology, 23, 1084-92.

Mousa N, Abdel-Razik A, Zaher A, et al (2016). The role of antioxidants and zinc in minimal hepatic encephalopathy: a randomized trial. Therap Adv Gastroenterol, 9, 684-91.

Padilla Ruiz MA (2016). Tablas de normalidad de la población en Cuba para los test psicométricos utilizados en el diagnóstico de la encefalopatía hepática mínima [Normality tables of the population in Cuba for the psychometric tests used in the diagnosis of minimal hepatic encephalopathy]. Rev Gastroenterol Peru, 36, 29-34.

Rathi S, Chopra M, Chouduri G, et al (2019). Prevalence of minimal hepatic encephalopathy in patients with liver cirrhosis: A cross-sectional, clinicoepidemiological, multicenter, nationwide study in India: The PREDICT Study. J Clin Exp Hepatol, 9, 476-83.

Riggio O, Ariosto F, Merli M, et al (1991). Short-term oral zinc supplementation does not improve chronic hepatic encephalopathy. Results of a double-blind crossover trial. Dig Dis Sci, 36, 1204-8.

Shawcross D, Jalan R (2005). The pathophysiologic basis of hepatic encephalopathy: central role for ammonia and inflammation. Cell Mol Life Sci, 62, 2295-304.

Shiha G, Mousa N (2019). Minimal hepatic encephalopathy: Silent tragedy. Liver Dis Surg, 2019.

Somi MH, Rezaeifar P, Ostad Rahimi A, et al (2012). Effects of low dose zinc supplementation on biochemical markers in non-alcoholic cirrhosis: a randomized clinical trial. Arch Iran Med, 15, 472-6.

Takuma Y, Nouso K, Makino Y, et al (2010). Clinical trial: oral zinc in hepatic encephalopathy. Aliment Pharmacol Ther, 32, 1080-90.

Urios A, Mangas-Losada A, Gimenez-Garzó C, et al (2017). Altered postural control and stability in cirrhotic patients with minimal hepatic encephalopathy correlate with cognitive deficits. Liver Int, 37, 1013-22.

Vilstrup H, Amodio P, Bajaj J, et al (2014). Hepatic encephalopathy in chronic liver disease: 2014 Practice Guideline by the American Association for the Study of Liver Diseases and the European Association for the Study of the Liver. Hepatology, 60, 715-35.

Ware JE, Kosinski M, Gandek B (2000): SF-36 ${ }^{\circledR}$ Health Survey: Manual \& Interpretation Guide. Lincoln, RI: QualityMetric Incorporated;1993.

Weissenborn K (2008). PHES: one label, different goods?!. J Hepatol. 49, 308-12.

Weissenborn K (2015). Diagnosis of minimal hepatic encephalopathy. J Clin Exp Hepatol, 5, 54-9.

Yanagisawa H (2008). Zinc deficiency and clinical practice-validity of zinc preparations. Yakugaku Zasshi, 128, 333-9.

\section{(2) $(1)$}

This work is licensed under a Creative Commons AttributionNon Commercial 4.0 International License. 